\title{
Doxycycline-Inducible Autoimmune Blistering Skin Disease Model
}

\author{
Wataru Nishie and Hiroshi Shimizu
}

\begin{abstract}
Bullous pemphigoid (BP) is the most common autoimmune blistering disorder. BP autoantibodies target two hemidesmosomal components, collagen XVII (COL17) and BP230, with autoimmunity to COL17 being mainly involved in the development of the disease. BP most commonly affects the elderly, and systemic corticosteroids are widely used to treat blisters on the entire body. Therefore, severe cases are sometimes fatal. Toward developing innovative, disease-specific therapies with fewer adverse effects, faithful disease models are essential. However, it has been challenging to reproduce BP in animals, because of inter-species differences in the amino acid sequences of pathological epitopes on human and mouse COL17. Human IgG autoantibodies from BP patients are unable to bind with mouse COL17; thus, the passive transfer of BP-IgG into mice fails to induce blistering disease. To overcome inter-species differences, we have generated genetically modified mice that express human but not mouse COL17 in skin. We call these "COL17-humanized mice". Using these mice, we have produced different BP models to elucidate the BP pathomechanism and to produce novel BP therapies. Currently, another novel model is under development. Here, we introduce the BP models that we have developed by using a novel technique called "humanization of autoantigen".
\end{abstract}

Keywords Autoimmunity $\bullet$ Collagen XVII • BP180 • Blistering disease $\bullet$ Skin $\bullet$ Humanized mouse

W. Nishie, M.D., Ph.D. • H. Shimizu, M.D., Ph.D. $(\bowtie)$

Department of Dermatology, Hokkaido University Graduate School of Medicine,

N15W7, Kita-ku, Sapporo 003-0835, Japan

e-mail: shimziu@med.hokudai.ac.jp

K. Nakao et al. (eds.), Innovative Medicine, DOI 10.1007/978-4-431-55651-0_9 


\section{Bullous Pemphigoid (BP), The Most Common Autoimmune Blistering Disease}

\section{Clinical Features of BP}

Bullous pemphigoid (BP) is the most common autoimmune blistering skin disorder, and the elderly are the most prone to it $[1,2]$. In typical cases of BP, urticarial erythemas with tense blisters on the trunk and extremities associated with pruritus are observed (Fig. 1a). BP usually develops around the seventh to eighth decades of life in both sexes. Individuals younger than age 50 rarely develop BP. Previous retrospective studies in European countries have shown a prevalence of around 6.6:1,000,000 per year, but recent analyses have shown an increased prevalence of 21.7 66:1,000,000 [3]. This increase is probably due to population aging and to development of diagnostic tools such as ELISA assays that can easily detect circulating autoantibodies (autoAbs).

\section{Pathogenesis of BP}

Direct immunofluorescence (IF) studies of the perilesional skin shows linear in vivo deposition of IgG and C3 at the dermal-epidermal junction (DEJ) (Fig. 1b), and circulating IgG autoAbs binding the DEJ of the skin can be found in sera from BP
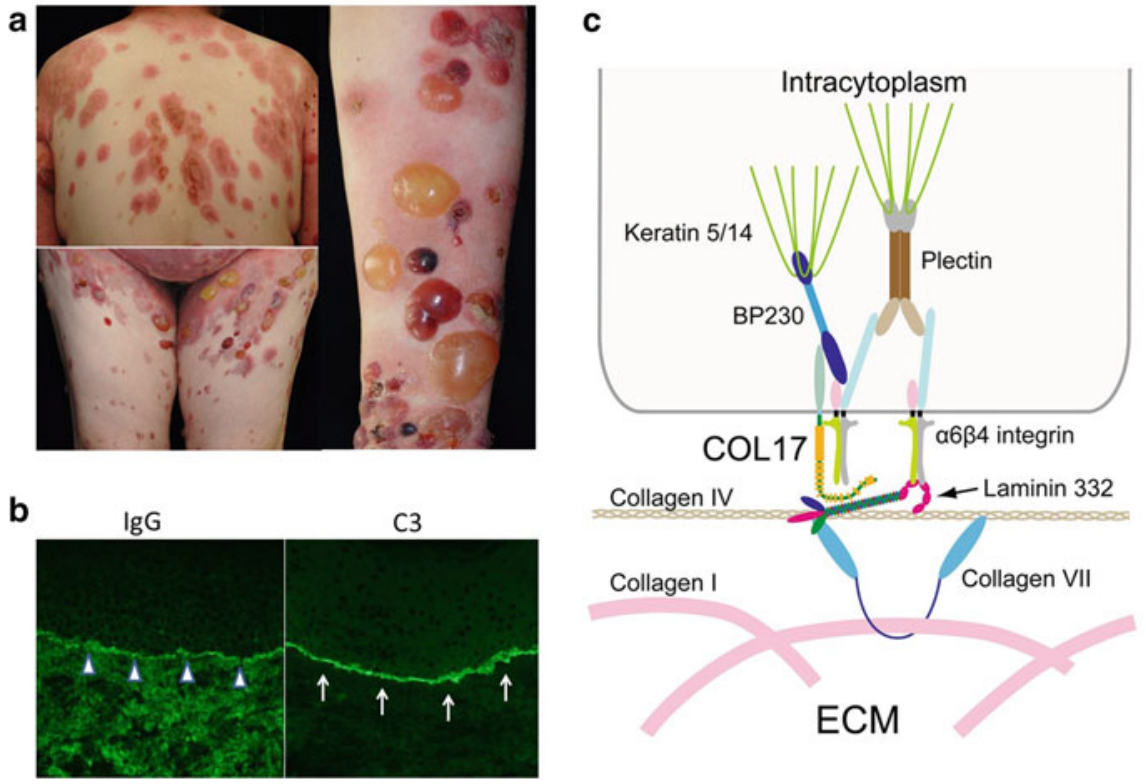

Fig. 1 Clinical and immunopathological manifestations of BP. (a) Urticarial erythema with tense blisters. (b) DIF studies of the lesional skin show linear in vivo deposition of IgG (arrowheads) and C3 (arrows) at the DEJ. (c) COL17 and hemidesmosomal molecules at the DEJ (partially modified from Nishie et al. [4]) 
patients by indirect IF. The autoAbs in BP patients target two hemidesmosomal components: transmembrane collagen XVII (COL17, also called BP180 or BPAG2) and the plakin family protein BP230 (BPAG1) (Fig. 1c). Of these, COL17 is thought to be the major autoantigen (autoAg) [4]. The IgG autoAbs against the COL17 in the $\mathrm{DEJ}$ of the skin are considered to trigger the inflammatory process, resulting in the disruption of dermal-epidermal anchoring [2, 3]. It remains uncertain as to whether anti-BP230 autoantibodies in BP patients are pathogenic for blister formation or whether they are just by-products of epitope-spreading associated with disease extension.

\section{COL17 as a Main AutoAg for BP}

COL17 is a type-II-oriented 1,497-amino-acid transmembrane collagen whose amino (N) terminus is in the cytoplasm and whose carboxyl (C) terminus is in the extracellular matrix (ECM) [5, 6]. As described in Fig. 1c, the extracellular domain of COL17 interacts with laminin 332 and collagen IV in the ECM, and the farthest part of the C-terminal region of COL17 has a flexible tail in vivo [7]. The extracellular domain of COL17 is constitutively cleaved (shed) from the cell surface by ADAM 9/10/17 [8, 9], a phenomenon that occurs within a juxtamembranous extracellular non-collagenous NC16A domain of 77 amino acids. Interestingly, the majority of IgG autoAbs from $\mathrm{BP}$ patients react with this NC16A domain, and epitopes cluster tightly in the $\mathrm{N}$-terminal 45 -amino-acid stretch of this region (Fig. 2a) [10, 11]. These findings indicate that autoAbs to the NC16A domain are pathogenic for blister formation in BP. However, human IgG autoantibodies from BP patients are unable to bind with mouse COL17; thus, the passive transfer of BP-IgG into mice fails to induce blistering disease [12]. This is attributed to the significant differences between human COL17 and mouse COL17 in amino acid sequences of the NC16A domain (Fig. 2a).

\section{BP Models Produced by "Humanization of Autoantigen"}

\section{Generation of COL17-Humanized Mice}

The pathological relevance of Abs to COL17 has been studied by neonatal mouse models that have been produced by injecting rabbit Abs targeting the NC14A domain of mouse COL17 (corresponding to NC16A domain of human COL17). When rabbit Abs targeting mouse COL17 were passively transferred into wild-type neonatal mice, the rabbit Abs bound with the DEJ of the mice skin, after which complement activation, mast cell degradation, neutrophil recruitment and, finally, epidermal detachment by mild friction occurred [12]. Using the experimental BP model mice induced by rabbit IgG against mouse COL17, complement activation followed by mast cell degradation and neutrophil infiltration have been suggested to be involved in the blister formation [13-15]. However, the pathogenic relevance of human autoAbs to COL17 in BP patients has never been proved. In 2007, our group 
a
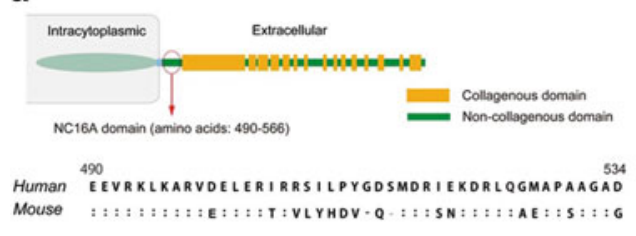

b

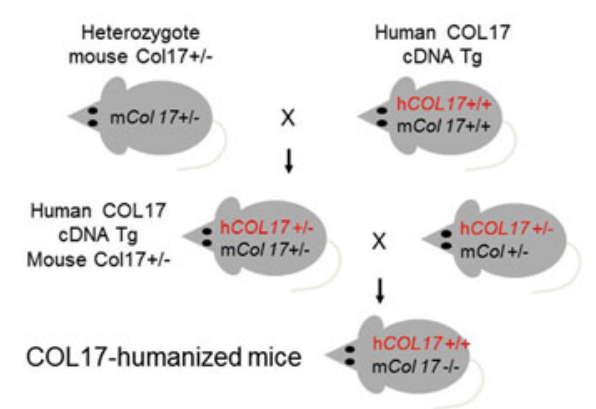

c

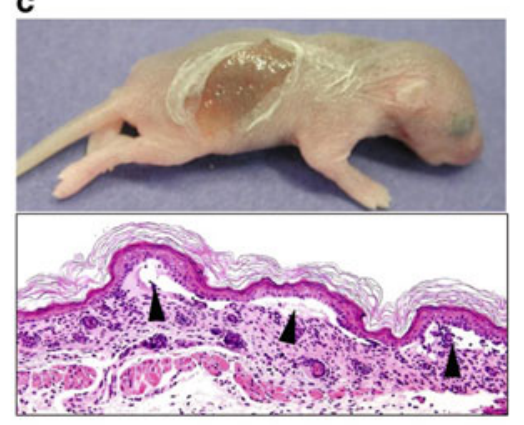

d

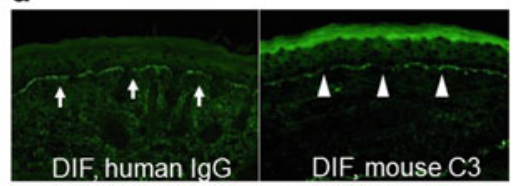

Fig. 2 Generation of the COL17-humanized mice and a BP model. (a) The most pathogenic of the epitopes cluster within the juxtamembranous NC16A domain of COL17. Significant differences in their amino acid sequences exist between the human and the mouse NC16A domains of COL17. (b) Generation of COL17-humanized mice by repeated crossing of heterozygous mouse Coll7knockout $\left(\mathrm{mCol17^{+/ }}\right)$ and human $\mathrm{COL17^{+/+ }} \mathrm{Tg}$ mice. (c) Skin detachment of the neonatal COL17humanized mice is inducible by mild friction $48 \mathrm{~h}$ after the injection of BP-IgG. (d) Histologically, subepidermal blister formation (arrowheads) associated with linear deposition of human IgG (arrows) and mouse C3 (white arrowheads) at the DEJ are observed

succeeded in confirming the pathogenicity of BP autoAbs by using the unique technique of "humanization of autoantigen" [4]. First, we generated murine COL17knockout $\left(\mathrm{mCol17^{-/ } )}\right.$ mice that developed blisters and erosions on the skin, symptoms that reproduce a human disease called non-Herlitz epidermolysis bullosa, which is caused by null mutations in the COL17Al gene [16]. Then, we crossed human $\mathrm{COL} 17^{+/+}\left(h \mathrm{COL} 17^{+/+}\right)$transgenic $(\mathrm{Tg})$ mice [17] with heterozygous $\mathrm{mCol17^{+/- }}$ mice. Mice that carried both the heterozygous null mutation of $\mathrm{mCol17}$ and the transgene of human $\mathrm{COL} 17\left(\mathrm{mCol17}^{+/-}, \mathrm{hCOL17^{+/- }}\right)$ were bred to generate COL17-humanized $\left(\mathrm{hCOL17^{+/+ }}, \mathrm{mColl1}^{-/-}\right)$mice (Fig. 2b). COL17-humanized mice lack mouse COL17 but express human COL17. They showed no apparent clinical phenotype and were able to deliver COL17-humanized mice pups by mating with COL17-humanized mice parents. Neonatal COL17-humanized mice that were passively transferred with either total IgG or IgG affinity-purified against the NC16A domain of COL17 from BP patients developed diffuse erythema and showed epidermal separation by gentle skin friction at $48 \mathrm{~h}$ after transfer (Fig. 2c) [4]. Lesional skin specimens demonstrated dermal-epidermal separation and inflammatory cell infiltration of neutrophils and lymphocytes. DIF analysis revealed linear deposition of human IgG and mouse $\mathrm{C} 3$ at the DEJ, which simulates the human BP phenotype (Fig. 2d). This passive-transfer neonatal mouse model was the first to directly show the pathogenicity of anti-COL17 NC16A IgG autoantibodies in BP patients. 
This COL17-humanized BP mouse model is quite valuable not only for elucidating the pathomechanisms of BP but also for innovating disease-specific therapies. We executed a therapeutic trial using human COL17 NC16A recombinant peptides. After the transfer of total IgG from BP patients, COL17-humanized mice were treated with a recombinant peptide composed of the NC16A domain. The treated mice showed markedly reduced blister formation [4]. In addition, we have developed a novel therapeutic strategy of treating BP by using the recombinant Fab fraction of IgG monoclonal antibodies to COL17 to block the complement activation that would otherwise be induced by pathogenic autoantibodies [18]. We used phage display to generate recombinant Fab fragments of $\mathrm{IgG}$ monoclonal antibodies against the human COL17 NC16A domain from antibody repertoires of BP patients. Two recombinant Fab fragments showed marked ability to inhibit the binding of BP autoantibodies and to inhibit subsequent complement activation in vitro. In the in vivo experiments using the COL17-humanized BP mouse model, these recombinant Fab fragments protected mice against BP-autoantibody-induced blistering disease [18]. Thus, the use of engineered Fab Abs to block pathogenic epitopes seems promising, and it may lead to disease-specific treatments for BP.

\section{Neonatal BP Model Induced by Maternally Transferred Abs to Human COL17}

Using the COL17-humanized mouse, we developed another neonatal BP mouse model, which was induced by maternally transferred antibodies. When heterozygous mouse COL17-deficient $\left(\mathrm{mColl}^{+/-}\right)$female mice are crossed with COL17humanized $\left(\mathrm{hCOL} 17^{+/+}, \mathrm{mCol1} 7^{-/-}\right)$male mice, it is expected that $50 \%$ of the pups will show the genotype of human $\mathrm{COL} 17^{+/-}$, mouse Col17 $7^{-1-}$. We immunized mouse $\mathrm{COL} 17^{+/-}$female mice with human COL17 and mated them with COL17-humanized $\left(\mathrm{hCOL} 17^{+/+}, \mathrm{mCol17^{-/- }}\right.$ ) male mice. As expected, the circulating anti-human COL17 $\mathrm{IgG}$ antibodies that were produced by the immunized mother were transferred into the neonates through not only the placenta but also the milk, and half of the neonates whose skin expressed human but not murine COL17 $\left(\mathrm{hCOL} 17^{+/-}, \mathrm{mCol17}^{-/-}\right)$ developed blisters associated with the histological and immunological features that are seen in BP patients [19]. This novel experimental system has some advantages: (1) the model does not require the technically difficult injection procedure; (2) the pathogenic IgG persists for longer than in conventional passive transfer models; and (3) the immune reaction totally depends on the murine immune system, even though the antigen is human COL17.

\section{Active BP Model}

To develop an active BP mouse model, we generated immunodeficient Rag$2^{-/-} /$COL17-humanized mice by crossing COL17-humanized mice with $R a g-2^{-/-}$ mice. As a next step, we isolated splenocytes from the wild-type mice immunized 
a

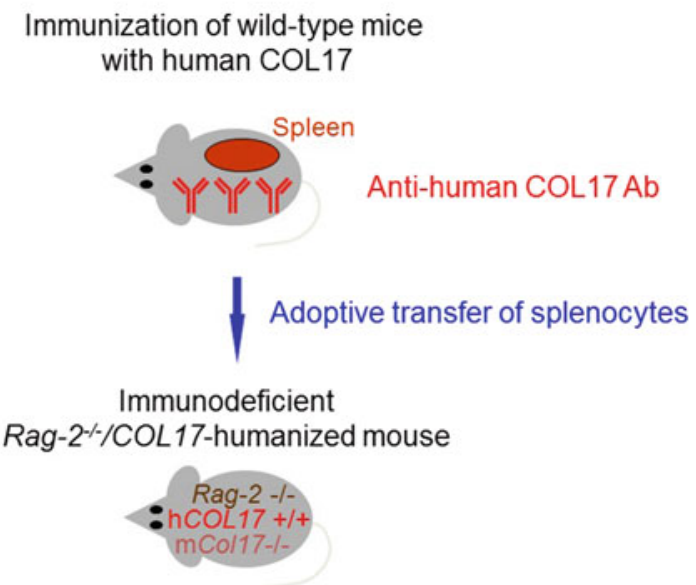

b

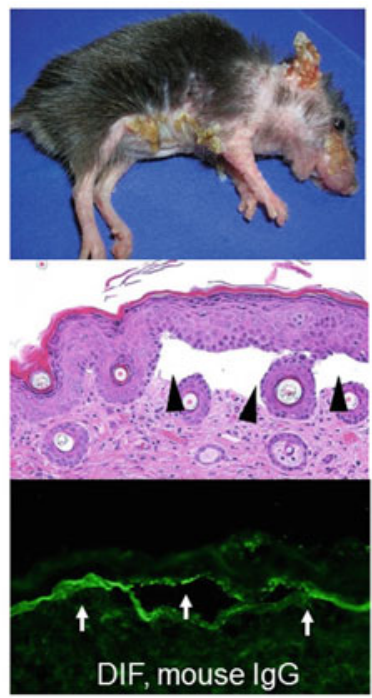

Fig. 3 Generation of an active BP model. (a) agg-2 $^{-/-}$COL17-humanized mice into which had been transferred splenocytes obtained from wild-type mice immunized by human COL17 protein. (b) The treatment induces the continuous production of anti-human COL17 IgG and blister formation corresponding to BP. Histologically, subepidermal blister formation (arrowheads) associated with linear deposition of mouse IgG (arrows) at the DEJ are observed

with human COL17 and adoptively transferred them into the $R a g-2^{-/-} /$COL17humanized mice. Because these Rag-2-/-/COL17-humanized mice had no mature T or B cells, they did not reject the transferred splenocytes. The transferred splenocytes induced continuous production of anti-human COL17 IgG, and blistering disease occurred (Fig. 3) [20]. In that study, CD4+ ${ }^{+}$cells were proven to be crucial for the development of the BP phenotype, because the depletion of $\mathrm{CD}^{+} \mathrm{T}$ cells from immunized splenocytes and the administration of cyclosporin A, a suppressant of T-cell function that works by inhibiting the production of IL-2 from the activated T cells, each significantly suppressed the development of pathogenic IgG and diminished the disease severity.

\section{Doxycycline-Inducible BP Model}

We have produced different BP models using the COL17-humanized mice as described. In these COL17-humanized mice, the innate expression of human COL17 eliminated all abnormal phenotypes observed in the Coll7-knockout (mColl ${ }^{-/-}$) mice [4]. If the expression of human COL17 is induced after birth in the Coll7knockout (mCol17 $\left.{ }^{-/}\right)$mice, what kinds of phenotypes will appear? Blistering diseases observed in adult Coll7-knockout $\left(\mathrm{mColl}^{-/-}\right)$mice may be rescued by 
neo-expression of human $\mathrm{COL} 17$ in the skin, but an immune reaction to human COL17 may occur. However, neo-expression of COL17 in adult Col17-knockout (mCol17 ${ }^{-/}$) mice may induce Abs to human COL17, leading to blistering disease similar to BP (Fig. 4a). We focused on using the Tet system that we commonly use to regulate expression of the gene of interest. By introducing transgenes including a reverse tetracycline-controlled transactivator (rtTA) and a pTRE vector with human COL17 cDNA, the administration of doxycycline induces the neo-expression of human COL17. In contrast, by incorporating a tetracycline-controlled transactivator (tTA) and a pTRE vector with human COL17 cDNA, the stable expression of human COL17 is expected to be suppressed by the administration of doxycycline. By in vitro studies using HEK 293 cells stably expressing pTRE-human COL17 and rtTA or tTA, we have found that the expression of human COL17 can be tightly regulated. In addition, the Tet-on system using rtTA showed less leaking expression of human COL17 (Fig. 4b). On the basis of these results, we started to prepare two

a
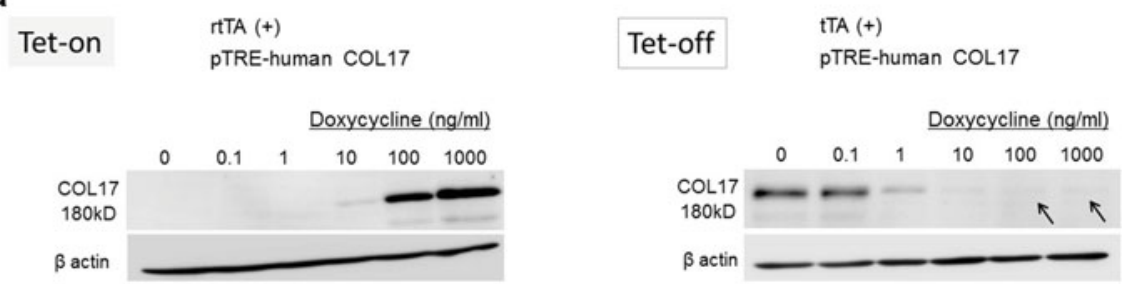

b

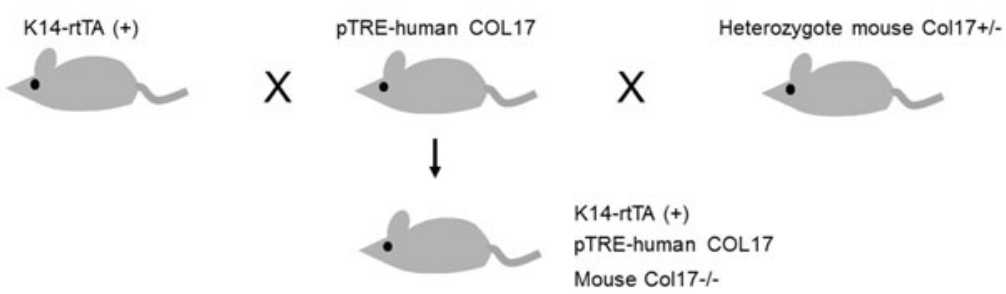

Oral doxycycline

Neo-expression of human COL17

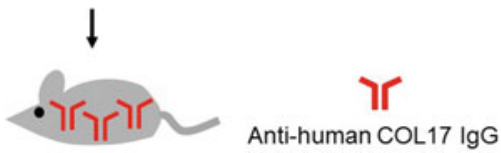

Fig. 4 Strategy for the generation of a doxycycline-inducible BP model. (a) In vitro studies using HEK 293 cells stably expressing pTRE-human COL17 and rtTA or tTA show that the expression of human COL17 can be tightly regulated. A Tet-on system using rtTA results in less leaking expression of human COL17 compared with a Tet-off system (arrows). (b) Oral administration of doxycycline to pTRE-COL17, K14-rtTA, mouse COL17-null mice is expected to induce neo-expression of human COL17 in basal epidermal keratinocytes, which would induce autoimmunity to human COL17 
different Tg mice expressing rtTA under keratin 14 (K14) promoter and pTREhuman COL17 cDNA. In the near future, we will obtain a novel BP model in which blistering disease can be initiated with only oral administration of doxycycline (Fig. 4a).

\section{Perspectives}

Recent studies using animal models have made considerable progress toward our understanding of the pathogenesis of BP as well as toward producing novel therapies. To further advance our knowledge of BP pathomechanisms and to develop novel therapeutic strategies for BP, the establishment of an active, stable disease model is anticipated.

Open Access This chapter is distributed under the terms of the Creative Commons Attribution Noncommercial License, which permits any noncommercial use, distribution, and reproduction in any medium, provided the original author(s) and source are credited.

\section{References}

1. Korman N (1987) Bullous pemphigoid. J Am Acad Dermatol 16(5 Pt 1):907-924

2. Nishie W (2014) Update on the pathogenesis of bullous pemphigoid: an autoantibodymediated blistering disease targeting collagen XVII. J Dermatol Sci 73(3):179-186. doi:10.1016/j.jdermsci.2013.12.001 S0923-1811(13)00386-1 [pii]

3. Schmidt E, Zillikens D (2013) Pemphigoid diseases. Lancet 381(9863):320-332. doi:10.1016/ S0140-6736(12)61140-4 S0140-6736(12)61140-4 [pii]

4. Nishie W, Sawamura D, Goto M, Ito K, Shibaki A, McMillan JR, Sakai K, Nakamura H, Olasz E, Yancey KB, Akiyama M, Shimizu H (2007) Humanization of autoantigen. Nat Med 13(3):378-383. doi:nm1496 [pii] 10.1038/nm1496

5. Diaz LA, Ratrie H 3rd, Saunders WS, Futamura S, Squiquera HL, Anhalt GJ, Giudice GJ (1990) Isolation of a human epidermal cDNA corresponding to the $180-\mathrm{kD}$ autoantigen recognized by bullous pemphigoid and herpes gestationis sera. Immunolocalization of this protein to the hemidesmosome. J Clin Invest 86(4):1088-1094. doi:10.1172/JCI114812

6. Giudice GJ, Squiquera HL, Elias PM, Diaz LA (1991) Identification of two collagen domains within the bullous pemphigoid autoantigen, BP180. J Clin Invest 87(2):734-738. doi:10.1172/ JCI115054

7. Nonaka S, Ishiko A, Masunaga T, Akiyama M, Owaribe K, Shimizu H, Nishikawa T (2000) The extracellular domain of BPAG2 has a loop structure in the carboxy terminal flexible tail in vivo. J Invest Dermatol 115(5):889-892. doi:jid136 [pii] 10.1046/j.1523-1747.2000.00136.x

8. Hirako Y, Usukura J, Uematsu J, Hashimoto T, Kitajima Y, Owaribe K (1998) Cleavage of BP180, a 180-kDa bullous pemphigoid antigen, yields a 120-kDa collagenous extracellular polypeptide. J Biol Chem 273(16):9711-9717

9. Franzke CW, Tasanen K, Schacke H, Zhou Z, Tryggvason K, Mauch C, Zigrino P, Sunnarborg S, Lee DC, Fahrenholz F, Bruckner-Tuderman L (2002) Transmembrane collagen XVII, an epithelial adhesion protein, is shed from the cell surface by ADAMs. EMBO J 21(19):5026-5035 
10. Zillikens D, Rose PA, Balding SD, Liu Z, Olague-Marchan M, Diaz LA, Giudice GJ (1997) Tight clustering of extracellular BP180 epitopes recognized by bullous pemphigoid autoantibodies. J Invest Dermatol 109(4):573-579. doi:S0022202X97890574 [pii]

11. Kobayashi M, Amagai M, Kuroda-Kinoshita K, Hashimoto T, Shirakata Y, Hashimoto K, Nishikawa T (2002) BP180 ELISA using bacterial recombinant NC16a protein as a diagnostic and monitoring tool for bullous pemphigoid. J Dermatol Sci 30(3):224-232. doi:S0923181102001093 [pii]

12. Liu Z, Diaz LA, Troy JL, Taylor AF, Emery DJ, Fairley JA, Giudice GJ (1993) A passive transfer model of the organ-specific autoimmune disease, bullous pemphigoid, using antibodies generated against the hemidesmosomal antigen, BP180. J Clin Invest 92(5):2480-2488. doi:10.1172/JCI116856

13. Liu Z, Giudice GJ, Swartz SJ, Fairley JA, Till GO, Troy JL, Diaz LA (1995) The role of complement in experimental bullous pemphigoid. J Clin Invest 95(4):1539-1544. doi:10.1172/JCI117826

14. Liu Z, Giudice GJ, Zhou X, Swartz SJ, Troy JL, Fairley JA, Till GO, Diaz LA (1997) A major role for neutrophils in experimental bullous pemphigoid. J Clin Invest 100(5):1256-1263. doi:10.1172/JCI119639

15. Liu Z, Shapiro SD, Zhou X, Twining SS, Senior RM, Giudice GJ, Fairley JA, Diaz LA (2000) A critical role for neutrophil elastase in experimental bullous pemphigoid. J Clin Invest 105(1):113-123. doi:10.1172/JCI3693

16. Gatalica B, Pulkkinen L, Li K, Kuokkanen K, Ryynanen M, McGrath JA, Uitto J (1997) Cloning of the human type XVII collagen gene (COL17A1), and detection of novel mutations in generalized atrophic benign epidermolysis bullosa. Am J Hum Genet 60(2):352-365

17. Olasz EB, Roh J, Yee CL, Arita K, Akiyama M, Shimizu H, Vogel JC, Yancey KB (2007) Human bullous pemphigoid antigen 2 transgenic skin elicits specific IgG in wild-type mice. $\mathrm{J}$ Invest Dermatol 127(12):2807-2817. doi:5700970 [pii] 10.1038/sj.jid.5700970

18. Wang G, Ujiie H, Shibaki A, Nishie W, Tateishi Y, Kikuchi K, Li Q, McMillan JR, Morioka H, Sawamura D, Nakamura H, Shimizu H (2010) Blockade of autoantibody-initiated tissue damage by using recombinant fab antibody fragments against pathogenic autoantigen. Am J Pathol 176(2):914-925. doi:10.2353/ajpath.2010.090744 S0002-9440(10)60403-8 [pii]

19. Nishie W, Sawamura D, Natsuga K, Shinkuma S, Goto M, Shibaki A, Ujiie H, Olasz E, Yancey KB, Shimizu H (2009) A novel humanized neonatal autoimmune blistering skin disease model induced by maternally transferred antibodies. J Immunol 183(6):4088-4093. doi:10.4049/jimmunol.0800389 jimmunol.0800389 [pii]

20. Ujiie H, Shibaki A, Nishie W, Sawamura D, Wang G, Tateishi Y, Li Q, Moriuchi R, Qiao H, Nakamura H, Akiyama M, Shimizu H (2010) A novel active mouse model for bullous pemphigoid targeting humanized pathogenic antigen. J Immunol 184(4):2166-2174. doi:10.4049/jimmunol.0903101 jimmunol.0903101 [pii] 